\section{BioMarin moves into muscular dystrophy with acquisition}

BioMarin Pharmaceuticals acquired the Dutch company Prosensa for $\$ 680$ million in December. Prosensa's lead product, an RNA therapeutic (exon-skipping) for muscular dystrophy, has a checkered past. Once part of a partnership arrangement with GlaxoSmithKline, the drug was returned to Prosensa in January 2014 after disappointing phase 3 clinical trial results. However, Prosensa revived the drug program in 2014 , and filed an new drug application with the US Food and Drug Administration (FDA) for accelerated approval in October with encouraging results in a small phase 2 trial. The FDA signaled its interest in the drug by granting it breakthrough drug status in 2013. BioMarin CEO was quoted as saying his company has experience with shepherding drugs that require "interpretation" through the regulatory process.

\section{PCORI takes up HCV treatment options}

The Patient-Centered Outcomes Research Institute (PCORI) announced in December its intent to put up to $\$ 50$ million into four comparative effectiveness research programs on hepatitis $C$ virus drug regimens. Two new and hugely expensive treatments approved in the recent past, with several more anticipated, may have sparked PCORI's interest. The main areas of interest, which emerged from a stakeholder meeting in October, are effectiveness of screening and diagnosis, head-to-head treatment comparisons and effect of different regimens for delivering care. The five-year-old agency, set up in accordance with the Affordable Care Act, will be undergoing a review of its own effectiveness in addressing its remit and its ability to evaluate its own work.

\begin{abstract}
C A judge [of the IGEM competition] asked the team whether designing a better diet pill was really the best way to combat obesity. The fact that you can create a biological shortcut, he noted, doesn't mean that you should." Nicola Twillley argues that not all problems should be addressed by technology. The judge was addressing a team from Hong Kong that had designed an $E$. coli to convert long-chain fatty acids to harmless unsaturated oil. (The New Yorker blog, 5 December 2014)
\end{abstract}

"Soon-Shiong may be a genius, but geniuses can be catastrophically wrong. That's why we demand proof. When it comes to SoonShiong's new efforts, we're still waiting." Matthew Herper takes a more jaundiced view of Shiong, billionaire entrepreneur, and his efforts to attack cancer, than the 60 Minutes' puff piece that aired on 7 December 2014. (Forbes, 7 December 2014) individuals, approximately $20 \%$ suffer from moderate to severe disease.

"The data we've shown with secukinumab show that up to $70 \%$ of patients can get to having clear or nearly clear skin," says Hohneker, including when it is the first drug they try.

"We do not need to move through a stepwise sequence of treatments before we get to one individual treatment," Bruce Strober of the University of Connecticut Health Center in Farmington told the advisory committee, speaking on behalf of Novartis. Even with the advent of the anti-TNFs, "psoriasis is undertreated," he said. Approximately 50\% of patients with moderate to severe psoriasis receive only topical or no therapy at all. And not surprisingly, he said, over half of the patients with moderate to severe disease are dissatisfied with current treatments.

According to one study, only $40-42 \%$ of patients stay on their original TNF blocker. And of the ones who didn't do well and had to change "The data in psoriasis clearly show that blocking IL-17 gives superior efficacy to anti-TNF." therapies, $37 \%$ of Enbrel patients and $45 \%$ of Humira (adalimumab) patients discontinued without restarting or switchingattributable to so-called 'biologic fatigue' where patients respond well at first, but then the biologic simply stops working or produces side effects that discourage compliance.

With both anti-TNFs and IL-17-blocking drugs, risk of infection remains an issue. Yeast infection in particular is a concern when blocking IL-17 because the cytokine is known to play a role in mucosal and skin host defense. Candida is also seen in patients who have IL-17 deficiency. With Cosentyx, candida infections were readily diagnosed and responded to standard topical or oral antifungal treatment, Novartis said in its presentation to the advisory committee on the drug. Neutropenia was more frequent in patients treated with Cosentyx and antiTNFs compared with placebo, which is expected because of the critical role IL-17 plays in neutrophil biology.

A limited number of observed adverse events, mostly infections, were present in patients and increased with higher exposure. That said, "safety does not seem to be a huge problem at this point," said committee chairperson Lynn Drake of Massachusetts General Hospital in Boston. However, she added, "everybody acknowledges that many of these signals don't come forward until the drug's out and about [for] a while" with mixed population in combination with other drugs. "I don't know that it would possible to get the long-term [safety] data that ideally we would like [in a clinical trial setting]," added biostatistician Erica Brittain of the National Institute of Allergy and Infectious Diseases.

"For certain diseases, I believe IL-17 has a unique role," says Wenjun Ouyang, of Genentech in S. San Francisco, California. "The data in psoriasis clearly shows that blocking IL-17 gives superior efficacy to anti-TNF," he says. But for other diseases, such as rheumatoid arthritis, the story might be more complicated. "There may be a segment of patients that may be responders to IL-17, especially those patients who do not respond to anti-TNF," he says. Genentech's antibody RG7624, which targets both the IL-17A and $\mathrm{F}$ subtypes, is in phase 1 testing in autoimmune diseases. There, as in other autoimmune diseases, development of a diagnostic strategy to identify patients who respond better to anti-TNF will be important, Ouyang says.

IL-17 is also associated with chronic inflammation and is linked to IL-23 and IL-6 in cancer. In preclinical cancer models, chronic inflammation pathways such as IL-23 and IL-6 pathways can promote colon cancer and liver cancer. IL-23 and IL-6 are upstream cytokines of IL-17 and T helper $17\left(\mathrm{~T}_{\mathrm{H}} 17\right)$ cells, which also have a role in the development of many autoimmune disorders including psoriasis, multiple sclerosis and rheumatoid arthritis.

Up to $30 \%$ of patients with psoriasis also have psoriatic arthritis (PsA). Patients with ankylosing spondylitis (AS) also often have skin manifestations. Novartis, Lilly and Amgen are all in phase 3 in PsA. Novartis is also in phase 3 in AS and intends to file for approval of Cosentyx in both PsA and AS in 2015.

Generally in autoimmune diseases, including PsA and AS, little has changed in the understanding of which patients are more likely responders to biologics targeting IL-17. But over the past several years an isoform of retinoic acid-related orphan receptor called ROR $\gamma t$, which is crucial for the development of $\left(\mathrm{T}_{\mathrm{H}} 17\right)$ cells, has piqued the interest of drug developers as another way to quiet IL-17.

Mark Ratner Boca Raton, Florida 